\title{
Manipulation de nanofils de silicium à partir de nano robots
}

\author{
J. P. Landesman ${ }^{\mathrm{a}, \mathrm{b}}$, L. Pichon ${ }^{\mathrm{a}, \mathrm{c}}$, F. Gouttefangeas ${ }^{\mathrm{d}}$ \\ ${ }^{\text {a}}$ Centre Commun de Microélectronique de l'Ouest, CCMO, Rennes \\ ${ }^{\mathrm{b}}$ Institut de Physique de Rennes, université de Rennes 1, UMR 6251, 35042 Rennes, France \\ 'Institut d'Electronique et de Télécommunications de Rennes,UMR 6164, 35042 Rennes \\ ${ }^{\mathrm{d} C M E B A-S c a n M A T}$, campus de beaulieu, université de Rennes 1 \\ Contact : lpichon@univ-rennes1.fr
}

\begin{abstract}
Nous présentons dans cet article une proposition de travaux pratiques sur la manipulation de nanofils de silicium individuels à partir de nanorobots pilotés par ordinateur. Ces nano-robots sont installés dans l'enceinte d'un microscope électronique à balayage. Le contrôle des nano-robots se fait par ordinateur via un module électronique d'interface.
\end{abstract}

\section{Introduction}

En raison de leurs propriétés physiques et électriques spécifiques, les nanofils de silicium font l'objet de nombreuses études grâce à leurs multiples applications potentielles dans l'élaboration de: - composants électroniques (nanotransistors) (1), - dispositifs photovoltaïques (2), - capteurs bio-chimiques (3), ou mécaniques MEMs (4).... En particulier, les nanofils de silicium peuvent être utilisés comme canal de conduction dans les transistors à effet de champ, ou encore comme unités sensibles dans les capteurs. De plus, les méthodes de synthèses des nanofils de silicium sont compatibles avec la technologie silicium existante, ce qui facilite leur intégration dans la fabrication de dispositifs électroniques ou de capteurs à faible coût de fabrication.

Il existe deux approches pour l'élaboration des nanofils de silicium : la voie «top-down » et la voie «bottom up ». Dans l'approche «top-down » divers outils lithographiques peuvent être utilisés : - électronique (5), - par oxydation locale du silicium (AFM) (6), - ou par insolation UV profonde (7). Bien qu'offrant une résolution à l'échelle nanométrique compatible avec la fabrication des nanofils, le principal inconvénient de ces outils repose sur leur coût élevé, et d'une manière générale ils sont incompatibles avec une production de masse. L'approche «bottom-up » quant à elle est basée sur la méthode d'auto-assemblage. Dans ce cas, la technique de synthèse la plus couramment utilisée est la méthode VLS (vapeur-liquide-solide), mettant en œuvre des nanoparticules métalliques utilisées comme catalyseur, associées à un gaz précurseur à base de silicium (8). Cette technique permet d'atteindre une production de masse des nanofils, tout en s'affranchissant de l'utilisation d'outils lithographiques onéreux. Cependant, la croissance 3-D ainsi que le contrôle de l'orientation des nanofils limitent leur intégration en technologie CMOS planaire. En effet, une méthode de report des nanofils est nécessaire dans ce cas.

La caractérisation directe d'un nanofil seul est problématique. Ainsi l'utilisation de nano manipulateurs est un moyen de cibler un nanofil individuel en vue de le caractériser, tout en s'affranchissant d'étapes technologiques complexes comme la réalisation d'électrodes adaptées pour une caractérisation électrique.

La manipulation de nanofils est d'un intérêt particulier dans le cadre d'études approfondies des propriétés physiques (thermiques, mécaniques...) et électriques en lien avec les structures et les défauts du matériau constituant le nanofil. Ainsi nous présentons ci-dessous une 
proposition de travaux pratiques basés sur la manipulation de nanofils de silicium à partir de nano-robots.

\section{Echantillons}

Les nanofils de silicium sont extraits de dispositifs à base de nanofils de silicium (fig. 1) fabriqués au Département Micro-électronique et Microcapteurs de 1'IETR. Ceux-ci sont constitués de pads à base de silicium polycristallin fortement dopé in situ au phosphore, déposé par procédé LPCVD (Low Pressure Chemical Vapor Deposition) sur un substrat (silicium ou verre) préalablement recouvert de $\mathrm{SiO}_{2}$. Une mince couche d'or $(<5 \mathrm{~nm})$ est ensuite déposée par évaporation thermique puis patternée par procédé lift-off sur les plots de silicium. La dernière étape consiste à faire croitre les nanofils par la méthode VLS à $460^{\circ} \mathrm{C}$ par procédé LPCVD. Les nanofils de silicium obtenus présentent un diamètre variant de 50 à $200 \mathrm{~nm}$. La durée de croissance des nanofils est choisie pour une obtention de nanofils d'une longueur suffisante $(\geq 20 \mu \mathrm{m})$.

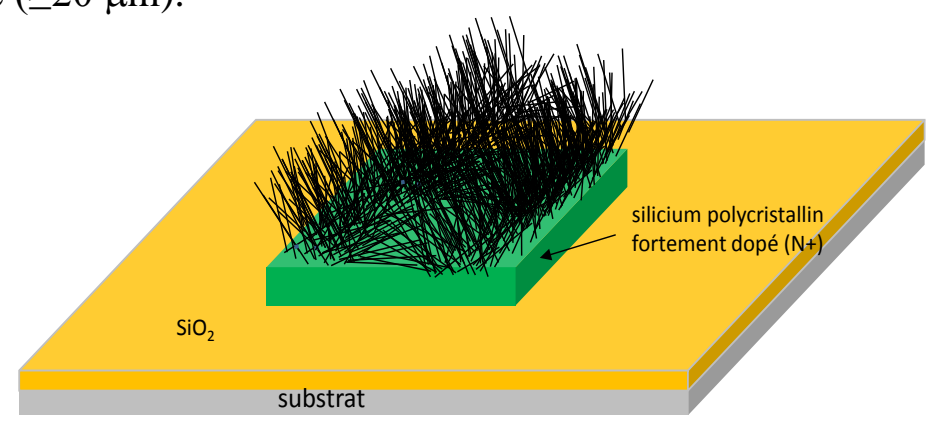

(a)

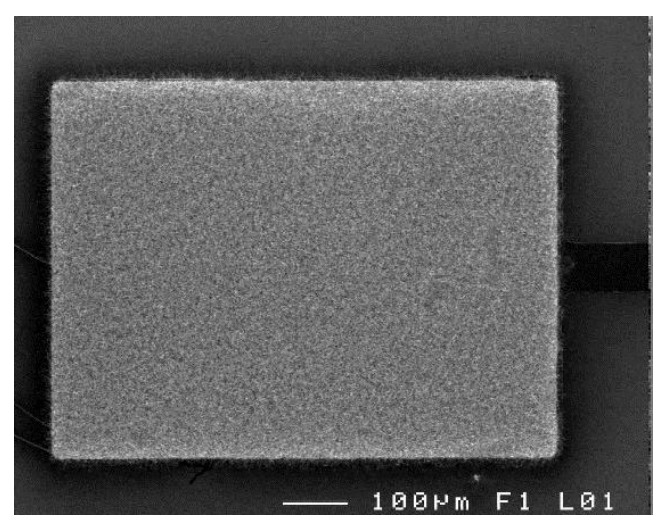

(b)

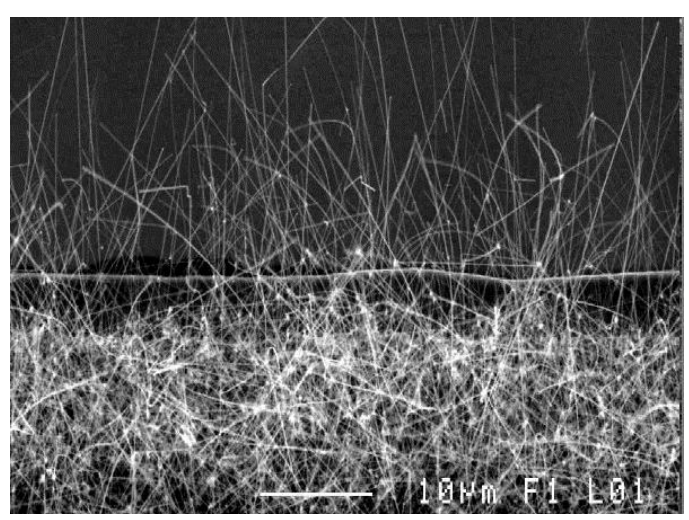

(c)

Fig.1 Vue schématique (a) et de l'image SEM d'un pad à base de SiNWs, (b) vue de dessus, (c) vue en coupe.

\section{Platine et nano-robots}

La platine ainsi que les quatre nano-robots (Fig.2) dont nous disposons ont été développés par la société IMINA Technologies. Cette platine a été spécialement conçue pour s'intégrer sur le porte objet d'un microscope électronique à balayage (JEOL modèle JSM-7100F TTLS) du «Centre de Microscopie Electronique à Balayage et microAnalyse » (CMEBA) de la plate-forme «Scan-MAT» l'université de Rennes 1». Chaque nano-robot présente une résolution de positionnement ajustable dans une grande gamme d'échelles (du micromètre au nanomètre), comprenant le câblage, les supports des nano-pointes, ainsi que le module électronique d'interface permettant le contrôle des robots par un ordinateur. La manipulation des nanofils se fait sous microscopie électronique à balayage (voir figure 3), dont une 
illustration est visible à partir de l'animation vidéo disponible sur le lien :

\section{畹)}

vlc-record-2017-04-04-08h27m14s-vlc-record-2017-04-04-08h20m40s-vlc-record-2017-04-03-18h47m06s-MVI_1603.MOV-.avi-.avi-.mp4

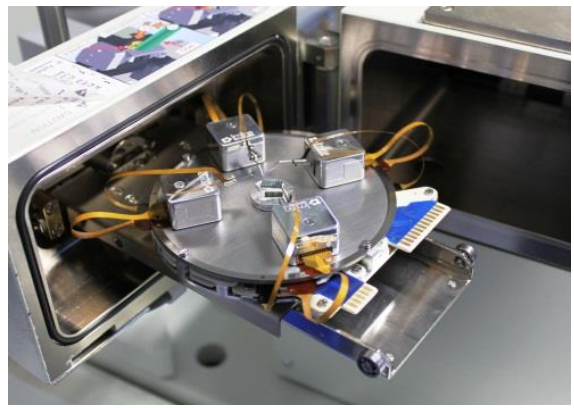

(a)

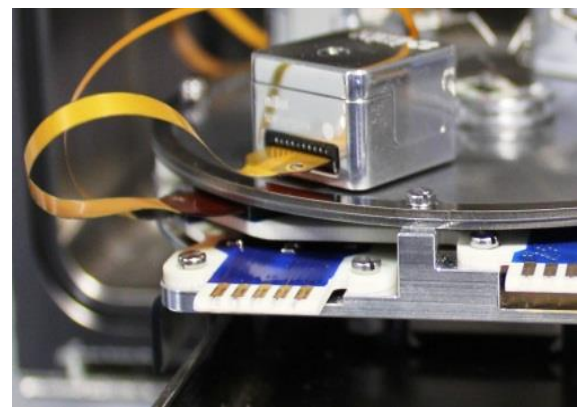

(b)

Fig. 2 : Platine et nano robots à l'entrée du sas du microscope électronique (a). La platine (amovible) est positionnée sur un support (fixe) sur lequel se trouve la connectique assurant le pilotage électrique des robots de chaque robot (b).
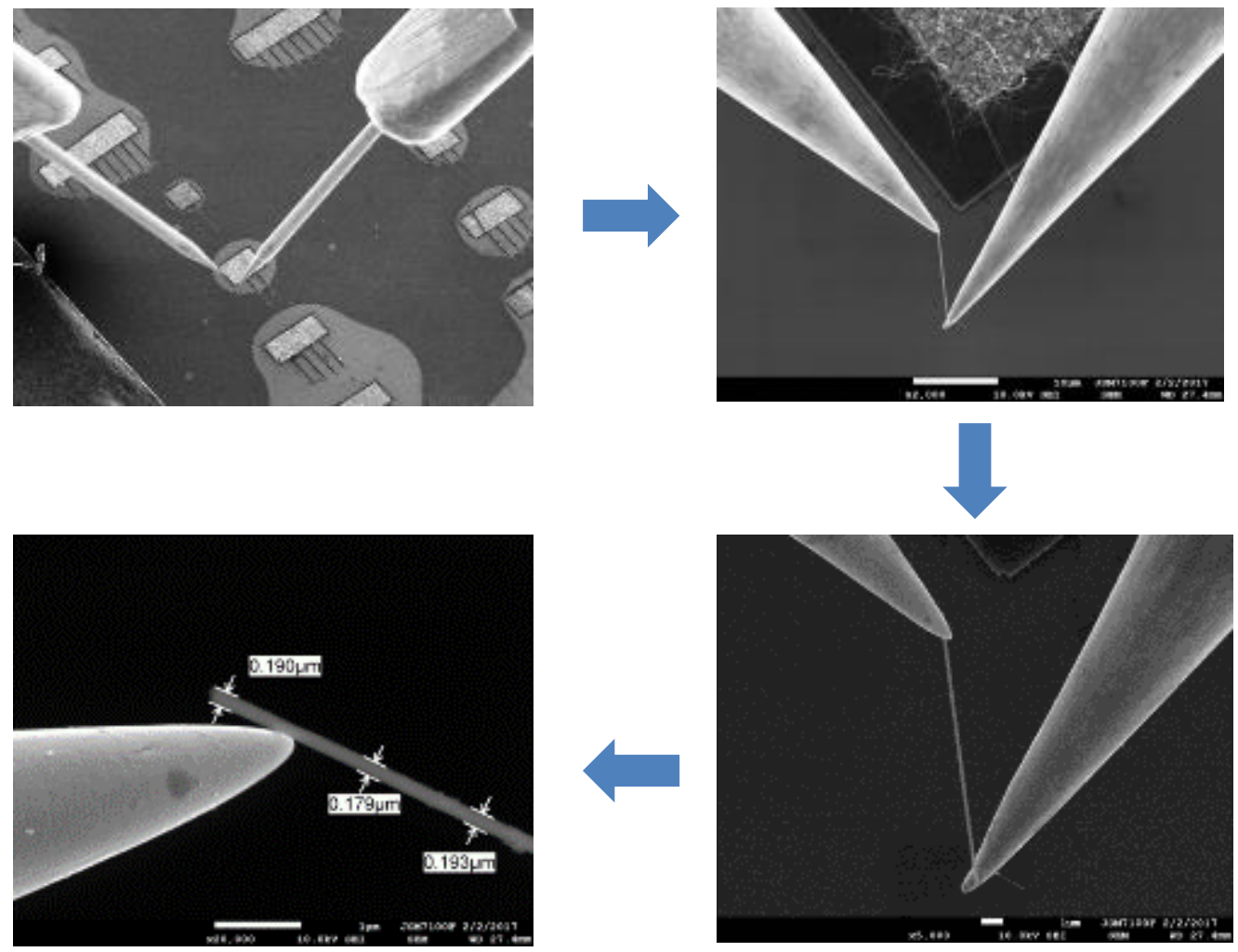

Fig. 3 : illustration de manipulation de nanofils. Accrochage sur les pointes des nano robots, transfert, puis mesures des dimensions nanofils.

La manipulation des robots se fait à partir d'une commande de type joystick identique à celle des jeux vidéos. L'ensemble platine + robots est démontable après chaque utilisation. Les pointes utilisées sont en tungstène, avec un rayon de courbure compris entre $100 \mathrm{~nm}$ et 20 $\mu \mathrm{m}$, en fonction des applications. Les 4 pointes sont connectées, via une connectique Triax, aux 4 entrées d'un source-mètre Keithley 2450. La connectique est optimisée pour les très faibles courants. Les objectifs recherchés de cet ensemble d'équipements sont : 
- être capable de repérer les nanofils, de les isoler puis de les saisir un à un et de les déplacer sur un substrat ad-hoc sur lequel des observations ou mesures peuvent être effectuées sur chaque nanofil,

- réaliser directement sur un nanofil unique des contacts électriques à l'aide des pointes métalliques qui sont approchées du nanofil grâce aux robots nano-manipulateurs. L'idéal sera d'amener 4 pointes au contact pour faire des mesures de conductivité électrique,

- contrôler le positionnement d'un nanofil isolé sur deux électrodes métalliques.

Un tel dispositif permet l'étude et la caractérisation d'un nanofil. Cet ensemble d'équipements pourra servir à la formation d'étudiants de Master. Cela reste aussi un outil performant pour les activités de recherche dans le domaine de la caractérisation des nano matériaux.

\section{Conclusion}

Cette proposition de TP qui est une initiation à la manipulation de nanofils de silicium individuels est un complément aux formations sur les «Micro et Nano-technologies» dispensées dans les masters ou filières d'écoles d'ingénieurs relevant des nanosciences. Les points forts du programme pédagogique abordés au cours de ce stage pratique concernent la synthèse des nanofils de silicium, la microscopie électronique à balayage, la caractérisation physique et électrique des nanofils en complément des enseignements de nanotechnologies, nano-caractérisation et de nanoélectronique.

\section{Remerciements}

La création de ce TP innovants a reçu le soutien du GIP-CNFM et du projet IDEFIFINMINA ANR 2011-IDFI-0017 dans le cadre du programme Grands Investissements d'Avenir (PIA1).

\section{Références}

1. J. Goldberger, A. I. Hochbaum, R. Fan, P. Yang, Silicon Vertically Integrated Nanowire Field Effect Transistors, Nanoletters, 6(5), 973 (2006)

2. T. J. Kempa, B. Tian, D. R. Kim, J. Hu, X. Zheng, and C. M. Lieber, Single and tandem axial p-i-n nanowire photovoltaic devices, Nanoletters, 8(10), 3456 (2008).

3. P. E. Nielsen, M. Egholm, R. H. Berg, O. Buchardt, Sequence-selective recognition of DNA by strand displacement with a thymine-substituted polyamide, Science, 254(5037), 1497 (1991).

4. Y. Cui, Q. Wei, H. K. Park, C. M. Lieber, Nanowire nanosensors for highly sensitive and selective detection of biological and chemical species Science 293(5533), 1289 (2001).

5. Z. Li, Y. Chen, X. Li, T. I. Kamins, K. Nauka, R. S. Williams, Sequence-specific label-free DNA sensors based on silicon nanowires Nanoletters, 4(2), 245 (2004)

6. I. Ionica, L. Montes, S. Ferraton, J. Zimmermann, L. Saminadayar and V. Bouchiat, Field effect and colomb blockage in silicon on insulator nanostructures fabricated by atomic force microscope Solid State Electronics, 49, 1497 (2005)

7. F. L. Yang, D. H. Lee, H. Y. Chen, C. Y. Chang, S. D. Liu and C. C. Huang, $5 \mathrm{~nm}$ gate nanowire FinFET, VLSI Symp. Tech. Dig. 196 (2004)

8. R.S. Wagner, W. C. Ellis, Vapor-Liquid-Solid mechanism of single crystal growth, Appl. Phys. Lett. 4(5), $89(1964)$ 